\title{
EL CSE Y LA ORIENTACIÓN VOCACIONAL. EXPERIENCIA DE ASISTENCIA TÉCNICA EN DOS LICEOS DE MAIPÚ Y PEÑALOLÉN
}

\section{Introducción}

La mirada panorámica del Consejo Superior de Educación (CSE) sobre el sistema de educación superior le ha permitido constatar que su masificación plantea importantes desafíos a la labor de orientación escolar. En el presente artículo se expone la evolución del trabajo del CSE respecto a la orientación vocacional, el que tuvo como foco inicial el apoyo a los orientadores de educación media y que, tanto a la luz de la experiencia como de la investigación desarrollada, se desplazó hacia la gestión escolar, vale decir, hacia un abordaje contextualizado de la orientación vocacional en el que la unidad de intervención y análisis son los liceos y no sólo los docentes que se desempeñan como orientadores.

Durante las últimas décadas nuestro país ha experimentado un proceso de creciente aumento en la cobertura del sistema de educación superior, entendido éste como un amplio conjunto de instituciones que abarca universidades, institutos profesionales, centros de formación técnica, además de las escuelas matrices y de especialidades vinculadas a las fuerzas armadas y de orden. Este aumento de cobertura e incipiente proceso de masificación ${ }^{2}$ se asocia no sólo con el surgimiento de universidades privadas, sino con una amplia oferta que incorpora diversos tipos de instituciones, así como con la disponibilidad de nuevas alternativas de apoyo económico para el financiamiento de carreras ${ }^{3}$.

2 Utilizando las proyecciones de población del Instituto Nacional de Estadísticas (INE) y las estadísticas de matrículas de la División de Educación Superior del Ministerio de Educación, hemos estimado que para 2007 la cobertura del Sistema de Educación Superior (SES) en Chile alcanzaba a un $38,4 \%$ de la población objetivo (18 a 24 años). De acuerdo con el modelo de Martin Trow (James, 2007), el SES de un país es considerado masivo cuando su cobertura se ubica en el rango que va entre el 16\% y $50 \%$ de la población objetivo.

3 Entre estas nuevas alternativas de apoyo económico podemos mencionar el Crédito con Aval del Estado, la Beca Bicentenario y la Beca Indígena. 
Dicho aumento en la cobertura ha permitido la incorporación al sistema de educación superior de muchos jóvenes que se constituyen en la primera generación de sus familias en acceder a estudios terciarios, es decir, jóvenes que no cuentan con referentes en sus grupos de socialización primaria que puedan orientar o apoyar su decisión de continuar estudios superiores.

Ante este escenario, el CSE, como organismo público con mirada sistémica que recopila y publica información panorámica y general acerca de la educación superior chilena, incorpora la orientación vocacional a su agenda de trabajo como parte de un continuo acercamiento al mundo de la educación escolar. No en vano una de las funciones relevantes del Consejo es pronunciarse sobre las propuestas de marcos curriculares y programas de estudio que elabora el Ministerio de Educación. A ello se agrega la larga trayectoria respecto de la generación de información relevante para el proceso de postulación de los egresados de secundaria a estudios superiores, especialmente las estadísticas de INDICES ${ }^{4}$.

Para narrar esta experiencia, en el primer apartado se explicará por qué el CSE incorporó en su agenda la problemática de la orientación vocacional y su acercamiento inicial al tema. En el segundo apartado se explicará el cambio de foco desde los orientadores a la gestión escolar de la orientación para, finalmente, en la tercera parte, informar sobre el proyecto de apoyo a la orientación implementado por el CSE a partir de 2008, materializado en la entrega de asistencia técnica a dos liceos municipalizados de la Región Metropolitana de Chile, uno en la comuna de Maipú y otro en la comuna de Peñalolén.

\section{Contexto e inicio de la experiencia: foco en orientadores, red nacional y foro}

La investigación educativa, tanto internacional como nacional, entrega pistas respecto de la relevancia y el potencial de los procesos de orientación vocacional desarrollados en el sistema escolar para

4 Base estadística realizada y publicada anualmente por el CSE. Para mayor información ver http://indices.cse.cl 
apoyar al tránsito de los estudiantes secundarios al mundo de los estudios superiores. Por una parte, se ha constatado que los procesos de orientación desarrollados en los establecimientos educacionales constituyen un instrumento potencialmente capaz de condicionar las elecciones académicas y profesionales de los estudiantes (Fernández, Peña, Viñuela y Torío, 2007) pudiendo incidir de manera decisiva en los procesos de tránsito entre un nivel educativo y el siguiente. Por otra parte, también existe evidencia en Chile respecto de que la carencia de una orientación vocacional adecuada sería uno de los factores explicativos de la deserción universitaria (De los Ríos y Canales, 2007).

Con el propósito de que la información generada por el CSE acerca del sistema de educación superior -especialmente INDICESllegara fácilmente a los estudiantes de educación media para que realizaran una postulación informada, se estableció un nexo directo con establecimientos de educación secundaria.

Fueron escogidos como interlocutores los orientadores de enseñanza media, en tanto actores influyentes en el proceso de selección de alternativas y postulación a la educación superior.

Una vez seleccionados los orientadores como foco de interés, se decidió concretar el nexo entre el CSE y dichos profesionales implementando una Red Nacional de Orientadores. Con ese objeto, se diseñó una base de datos con orientadores de todo el país, la que fue incrementando su número en paralelo a la instalación de la red. Un hito importante en este proceso fue la circulación de un documento de apoyo vocacional en conjunto con la publicación impresa de INDICES de 2005. En este periodo se mantuvo contacto mediante correo electrónico con los orientadores que se incorporaban al registro y, a poco andar, se afinaron los propósitos de esta línea de acción definiendo como objetivos generales:

- Establecer coordinación con otras instancias de organización en torno a los orientadores de enseñanza media.

- Habilitar un espacio de interacción virtual a través del cual materializar el funcionamiento de la red como tal, es decir, una instancia en la que se combinan el conocimiento, la comunicación 
y la colaboración entre los orientadores, desde la cual deberían surgir efectos positivos para el tránsito de los estudiantes secundarios al mundo de los estudios superiores.

El primer objetivo se concretó estableciendo un vínculo de colaboración con la línea de orientación vocacional del programa "Chilecalifica", la que venía operando desde 2003, utilizando como estrategia el desarrollo de redes de orientadores locales, principalmente comunales. Dicha colaboración se materializó, principalmente, a través de la organización de talleres conjuntos con orientadores en las ciudades de Santiago, Concepción, Viña del Mar y La Serena, en los que un representante de la Secretaría Técnica (Sectec) del CSE entregó información general acerca de la institucionalidad y las características del sistema de educación superior, y formación destinada a una habilitación de los integrantes de la red en el uso de INDICES.

La estrategia implementada para el logro del segundo objetivo fue el desarrollo de un sitio web (http://orientadores.cse.cl) que sirviera para mantener la comunicación interna entre los integrantes de la potencial red (diálogo entre orientadores y comunicación con el CSE), la difusión de noticias relevantes sobre el campo de la educación superior y la orientación vocacional, como también el intercambio de documentos técnicos publicados por el CSE o bien por los mismos orientadores.

En general, estas medidas tuvieron efectos en tanto fueron animadas directamente desde el CSE durante el periodo en que se realizaron los talleres para dar a conocer INDICES. Con todo, en junio de 2007 la red llegó a contar con 824 orientadores registrados y la página se mantuvo actualizada con noticias y documentos de apoyo técnico.

El cuanto al segundo objetivo, esto es, que los orientadores registrados lograran conocerse, comunicarse entre sí y finalmente

5 El sistema de formación permanente "Chilecalifica" es un programa articulado por los ministerios de Economía, Trabajo y Educación del Estado de Chile, surgido en 2002, con el propósito de mejorar la equidad de oportunidades, mejorando la competitividad y las condiciones de la fuerza laboral chilena. Este programa cuenta con una línea de trabajo de orientación vocacional y laboral, la cual comenzó a ser implementada en 2003. 
establecer una relación de colaboración técnica con vistas a optimizar el tránsito a estudios superiores de los estudiantes a su cargo, no se obtuvieron los resultados esperados.

En efecto, el funcionamiento del foro estuvo marcado por su carácter unidireccional, animado solamente por su administrador del CSE, lo que en la práctica derivó en que este espacio se convirtiera en un repositorio de documentos técnicos sobre orientación, alimentado casi exclusivamente por el CSE.

Así, la actitud de los orientadores fue más bien pasiva y sus visitas al Foro fueron estacionales, concentrándose en el periodo de postulación. Para ilustrar esta situación podemos citar el Informe de gestión de Gobierno Electrónico para el año 2007 del CSE, en el cual se indica que: "si bien el $48 \%$ de cobertura de colegios participantes en la red estuvo muy próximo al 50\% de cobertura, que se instauró como meta, no aconteció lo mismo con los niveles de participación esperado. En este ámbito, se ha presentado una actitud más pasiva, que constituye un desafío para enfrentar y asegurar la sustantividad y éxito de este proyecto" (CSE, 2007:112).

Estos problemas en ningún caso justifican desechar de manera definitiva el concepto de red como una herramienta de trabajo con interesante potencial. La evaluación al interior del CSE da cuenta de algunos errores en la implementación que pueden servir como aprendizaje para futuros intentos de trabajo con un soporte informático, ya sea desde el mismo Consejo o bien en otras instituciones vinculadas al campo de la educación:

En primer lugar, la poca experiencia del equipo a cargo en proyectos de intervención escolar con uso de Tecnologías de la Información y Comunicación (TICs). El soporte computacional con el que operó el foro no era de fácil acceso para los usuarios, prueba de ello es que, en junio de 2008, se debió diseñar y distribuir un manual de usuario para que los orientadores pudieran acceder a él. La estacionalidad del uso y lo engorroso del acceso al sitio web

6 Informe de gestión "Gobierno Electrónico 2007, Etapas I, II, II, IV y V reducida", Secretaría Técnica del Consejo Superior de Educación, 2007. 
provocó que varios orientadores, que mostraron interés en acceder a información publicada durante 2008, no pudieran entrar al sitio por problemas con sus claves. De lo anterior podemos concluir que una experiencia de este tipo requiere un background relevante acerca del trabajo de comunidades de aprendizaje digital.

Otro obstáculo para la implementación exitosa de este proyecto de red fue que no se tomaron en cuenta debidamente las condiciones de precariedad material que enfrentan muchos de los orientadores de los establecimientos educacionales del sector municipal.

Los dos puntos anteriores responden a errores de implementación que obstaculizaron la conformación exitosa de una red de orientadores. Sin embargo, la investigación realizada por el CSE en el mismo periodo en que se desarrollaba la iniciativa de formación de redes aporta algunas luces acerca de las dificultades estructurales que afectan el quehacer actual de los orientadores. Como se verá en el siguiente apartado, dichos hallazgos de investigación y la evaluación autocrítica de la estrategia implementada hasta ese punto son los elementos que justificaron el cambio de foco desde los orientadores hacia la orientación, como un proceso de gestión escolar.

\section{Del foco en los orientadores al foco en la orientación}

\section{1 ¿Por qué la insuficiencia del foco en los orientadores?}

En paralelo, durante el primer semestre de 2007 los investigadores adscritos a la Secretaría Técnica del CSE, Felipe Lagos y Fernanda Palacios, desarrollaron el estudio "Orientación vocacional y profesional en colegios de bajo nivel socioeconómico: percepciones de orientadores y estudiantes", el que entrega pistas que apuntan a la situación estructural de la orientación vocacional en los liceos municipales como principal limitante del accionar de los orientadores, al punto que, para mejorar su situación, no basta sólo trabajar con ellos, sino que intervenir en la gestión de los establecimientos, de manera que ésta incorpore a la orientación como un aspecto relevante y permanente del funcionamiento de los liceos. 
El estudio de Lagos y Palacios (2008) entrega una visión general del escenario al que se enfrentan actualmente los orientadores vocacionales de enseñanza media, destacando entre otros problemas:

La inexistencia de una normativa concreta que regule el marco de acción del orientador. La derogada circular $\mathrm{N}^{\circ} 600$ del MINEDUC (1991) proponía que los establecimientos elaboraran su propio "plan anual de orientación", estipulaba que la orientación debía estar a cargo de un profesional con postítulo de consejero educacional y vocacional $\mathrm{u}$ orientador en una universidad reconocida por el Estado, y llamaba a que cada establecimiento, básico completo o de enseñanza media, contara con, al menos, un orientador con una carga horaria pertinente. En su reemplazo no se incorporó un equivalente funcional, ya que el Decreto $N^{\circ} 220$ del MINEDUC (1998) establece que en enseñanza media la orientación pasa a ser transversal a la educación, por lo que en la práctica queda sin un espacio curricular específico.

Escasean las instancias de conocimiento entre el orientador y los alumnos, lo que se asocia a la desequilibrada proporción orientadores/ alumnos (1/600) y a la práctica extendida de privilegiar la atención directa que, dadas las constricciones de tiempo, implica una cobertura limitada en términos numéricos.

Hay pocas instancias de coordinación entre docentes para definir estrategias comunes, lo que, nuevamente, se asocia a la atención directa e individual a los estudiantes. Los orientadores están colapsados con la atención de alumnos y no cuentan con espacios para coordinar estrategias con los demás docentes del establecimiento.

Estos antecedentes sugieren que los orientadores no disponen del espacio institucional, ni del tiempo pertinente para coordinar y ejecutar acciones articuladas en las que participen más actores de la comunidad educativa.

Entre las conclusiones del estudio citado se encuentran antecedentes que permiten inferir que, en el actual escenario educacional, los orientadores tienen un margen de acción escaso, 
tanto por la carencia de un marco normativo adecuado como por el carácter difuso y disperso de su función:

- Por un lado, los propios estudiantes indican que la labor de los orientadores tendría mayor impacto si éstos se hicieran cargo de menos temas o fueran apoyados por un equipo más amplio.

- La multiplicidad de funciones afecta en parte a la orientación vocacional profesional porque la marginaliza y, a la larga, también afecta a las restantes funciones asociadas tradicionalmente a la labor de los orientadores, ya que la dispersión no les permite esfuerzos concentrados en ninguna dimensión.

En síntesis, la ausencia de una normativa clara, la multiplicidad de labores y las precariedades propias de los establecimientos que operan en contextos de carencias impactan de manera importante en la efectividad de la orientación vocacional; factores que, en conjunto, ayudarían a explicar por qué las tareas específicas de orientación vocacional se ven desplazadas a momentos puntuales del calendario escolar, como la inscripción en pruebas de selección nacional o las postulaciones.

Estos antecedentes empíricos indican que, para poder intervenir de manera efectiva en el sistema escolar, facilitando el tránsito de los estudiantes entre la educación secundaria y los estudios superiores, no basta con un enfoque limitado exclusivamente a los orientadores, ya que las condiciones en que actualmente desempeñan sus funciones limitan significativamente su capacidad de acción.

Por esta razón, durante el segundo semestre de 2007 la Sectec del CSE decidió ampliar el enfoque para la problemática de la orientación, asumiendo que, para lograr mejoras sustantivas en esta labor y para que ésta contribuya efectivamente a cerrar, o al menos reducir, las brechas de acceso a información relevante para la postulación a estudios superiores, es necesario incorporar a la orientación como un componente central de la gestión escolar.

Bajo esta convicción, el enfoque del problema se desplaza de los orientadores en tanto actores, a la orientación en tanto proceso relevante de la gestión escolar, en el cual no sólo participan 
orientadores, sino que también directivos y otros docentes. Al abordar la orientación como parte de un despliegue de acciones intencionadas e inspiradas en un horizonte estratégico integral, su despliegue no sólo se concentra en ciertos periodos puntuales del año, sino que tiene un papel preponderante a lo largo de toda la programación anual.

En consecuencia, el foco de intervención y observación ya no son los orientadores, sino que los establecimientos educacionales o liceos en su totalidad.

\subsection{El foco en los liceos para mejorar los procesos de orientación: encuadre conceptual de una opción}

Al revisar bibliografía producida en Chile respecto de la mejora de procesos educacionales, encontramos que Raczynski y Muñoz (2007) distinguen claramente dos grupos de factores que inciden en la calidad y la equidad educativa:

a) Aquellos que dependen casi exclusivamente de las unidades educativas (escuelas o liceos).

b) Los factores exógenos, tradicionalmente conocidos como "variables estructurales".

Los primeros corresponden a las variables que reflejan el modo en que la escuela enfrenta el desafío de entregar un buen servicio educativo a sus estudiantes. Los autores citados aluden a la gestión interna, el trabajo docente en la sala de clases y la organización del tiempo escolar.

Como parte del segundo grupo de factores -variables estructurales-, mencionan la formación inicial docente, la estructura administrativa, el marco regulatorio que rige al sistema y la modalidad de financiamiento.

Considerando el diagnóstico antes expuesto, en el escenario actual de la orientación vocacional en liceos con estudiantes de sectores populares se observan dificultades en las dos categorías mencionadas; la ausencia de una normativa clara respecto a la orientación aparece claramente como una variable exógena o estructural, mientras que la multiplicidad de funciones por parte de los orientadores y la falta 
de instancias de coordinación que conllevan a la marginalización de la función de apoyo a la orientación vocacional pueden catalogarse como factores más asociados a la unidad educativa en sí, es decir, como factores de gestión escolar.

La incorporación de la orientación como un componente relevante de la gestión escolar ha sido abordada por otros entes públicos, como "Chilecalifica" y el Ministerio de Educación (MINEDUC), entidades que en un documento conjunto plantean que la concepción de un modelo de orientación educacional eficaz "no es posible sino en el marco de una participación activa de la orientación en la gestión directiva de los liceos. De modo que ésta se asuma como responsabilidad del conjunto de los actores del sistema y el orientador juegue un papel activo en el diseño y seguimiento del $\mathrm{PEI}^{7}$ y en la asesoría directa y permanente a los docentes en sus estrategias pedagógicas" (Chilecalifica, MINEDUC, 2008).

Dado que el CSE no cuenta con facultades para alterar el marco normativo que regula la orientación vocacional en los liceos de Chile, la opción más viable para incidir en la mejora de los procesos de orientación vocacional es intervenir en aquellas variables que son manejables desde las unidades educativas mismas, vale decir, en la gestión escolar.

\section{Proyecto de apoyo a la orientación CSE 2008}

Como ya se señaló, la experiencia acumulada durante la implementación de la red nacional y el foro de orientadores del CSE, así como la investigación empírica realizada en paralelo, fundamentaron la decisión de un cambio de estrategia, desplazando el foco desde los orientadores a los procesos de orientación en la gestión escolar. Para la implementación de esta nueva estrategia se optó por realizar una intervención acotada a un par de establecimientos, que además permitiera profundizar en el conocimiento de la realidad en que se desenvuelve la labor de orientación, ya entendida en el contexto de la gestión escolar. Se trata de un proyecto de asistencia técnica que

7 Proyecto Educativo Institucional. 
tuvo su primer año de ejecución durante 2008 en las comunas de Maipú y Peñalolén.

En la misma línea de trabajo se han generado dos proyectos de investigación, el ya citado de Lagos y Palacios (2008), y un estudio de seguimiento a egresados de secundaria cuyos resultados estarán disponibles el segundo semestre de 2009. Sin embargo, el foco del presente documento es la experiencia de asistencia técnica que a continuación se relata.

\subsection{Antecedentes generales}

Entre enero y marzo de 2008, el CSE tomó contacto con dos corporaciones municipales de educación, la CORMUP de Peñalolén y la CODEDUC de Maipú, con el fin de solicitar autorización para desarrollar este proyecto de asistencia técnica, en conjunto con liceos de su dependencia. La selección de estas comunas se realizó en función del perfil pensado para los liceos con los que se trabajaría: del segmento socioeconómico medio-bajo, según la clasificación del MINEDUC.

Actualmente, el proyecto se está implementando en los liceos "Municipal de Maipú, Alcalde Gonzalo Pérez Llona" y "Antonio Hermida Fabres" de Peñalolén, establecimientos seleccionados de común acuerdo con ambas corporaciones.

Al seleccionar este segmento socioeconómico se busca introducir mejoras en la orientación vocacional que reciben aquellos sectores sociales de incorporación reciente al sistema de educación superior, dada la creciente masificación de este último. Estos sectores serían los más afectados por las desigualdades de distribución y socialización del capital informacional respecto a la educación postsecundaria, debido a que están compuestos por estudiantes provenientes de familias que accederán por primera generación a ésta y que cuentan con menor capital social y cultural para orientar su selección, lo cual los convierte en un nicho de intervención pertinente. Esta situación ha sido documentada por la investigación internacional, a través de estudiosos como Patricia M. McDonough, en su libro "Choosing Colleges: How Social Class and Schools Structure Opportunity". La investigadora norteamericana señala que: "los 
estudiantes que corresponden a la primera generación de su familia en acceder al college, comienzan a pensar acerca de asistir al college mucho más tarde que aquellos estudiantes cuyos padres han accedido al college, y sus ideas al respecto son gatilladas por el personal de sus escuelas, específicamente profesores y orientadores. Los estudiantes cuyos padres han asistido al collage, frecuentemente comienzan su preparación para éste en la escuela primaria, tomando los cursos correctos y manteniendo buenas calificaciones, además sus familias les han transmitido información acerca de los diferentes tipos de colleges y universidades" (McDonough, 1997: 6) ${ }^{8}$.

Una encuesta realizada por el equipo del CSE durante abril de 2008 a los apoderados de tercero y cuarto medio de ambos liceos, pone en evidencia que la gran mayoría de ellos no ha tenido acceso a estudios superiores, al punto que en Peñalolén un importante porcentaje de apoderados no completó la educación básica. Por esta razón, el papel de los profesores y docentes como referentes para la prosecución de estudios superiores es de suma relevancia:

Gráfico $\mathrm{N}^{\circ} 1$. Nivel educacional de los apoderados de $3^{\circ}$ y $4^{\circ}$ año medio. Liceo Antonio Hermida de Peñalolén (base: 56 apoderados).

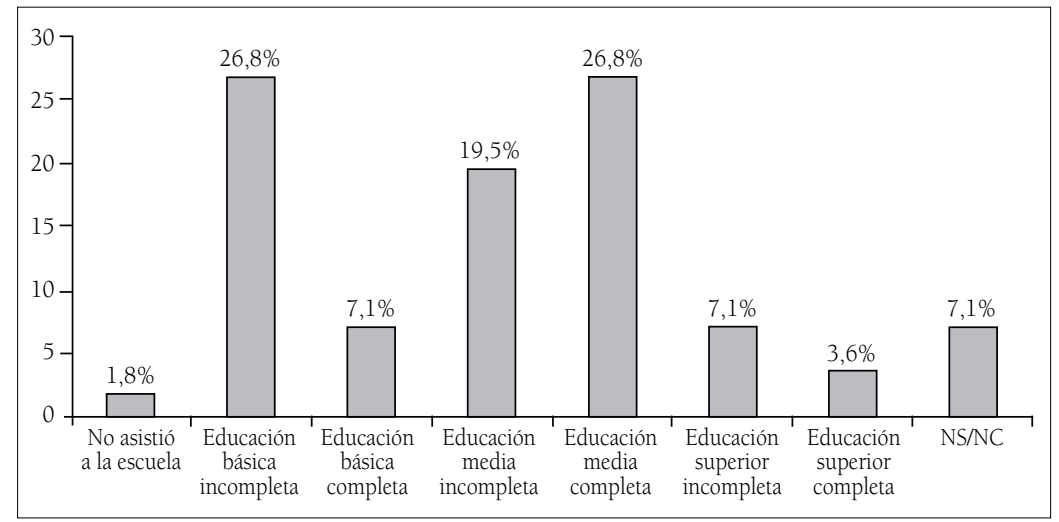

8 "Students who are first-generation college-bound begin to think about going to college much later tan do students whose parents have gone to college, and those thoughts tend to be triggered by school personnel, specifically teachers and counselors. Students whose parents have gone to college often get a head start on college preparation in elementary school by taking the right courses and maintaining good grades, and their families convey information to them about the different type of colleges and universities". 
Gráfico $N^{\circ}$ 2. Nivel educacional de los apoderados de $3^{\circ}$ y $4^{\circ}$ año. Liceo Municipal de Maipú (base: 108 apoderados).

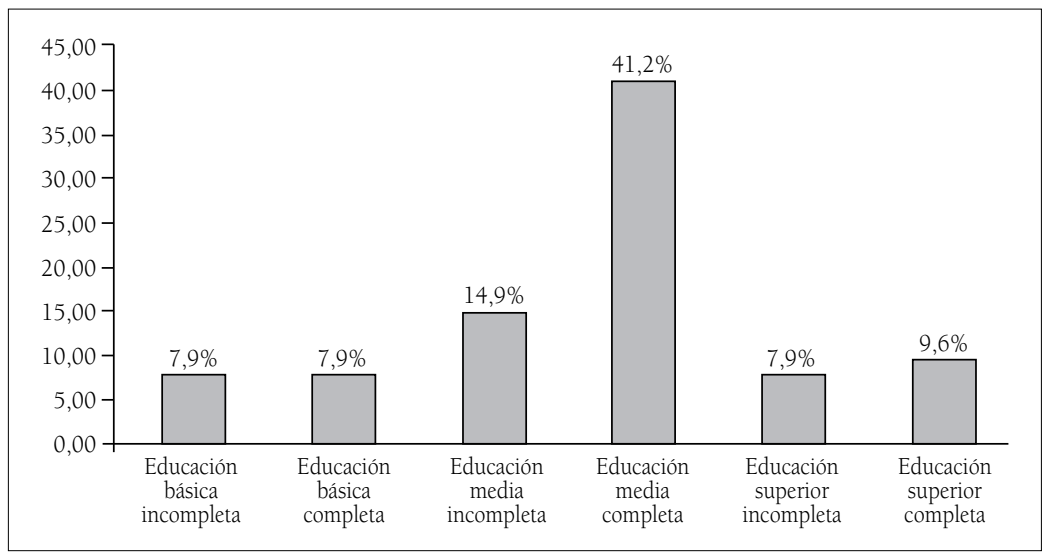

Esta selección no considera al estrato socioeconómico bajo (clasificación MINEDUC), por tratarse de un sector en el que las prioridades para la orientación se concentran en temas como la retención, problema que requiere, en alguna medida, tenerlo bajo control antes de instalar el de tránsito a la vida postsecundaria como una preocupación visible para la gestión escolar. A su vez, se optó por no trabajar con establecimientos de segmentos más altos, por considerar que éstos se encontrarían en una situación ventajosa en lo que a capital social y cultural se refiere.

\subsection{Diagnóstico y definición de objetivos generales para el proyecto de asistencia técnica}

A la luz de la investigación, la experiencia anterior y, principalmente, de la inserción inicial en los dos establecimientos, se llegó al siguiente diagnóstico:

- La multiplicidad de funciones de los orientadores y la exclusión de la temática vocacional del currículo escolar han derivado en la figura de un orientador sobrecargado de labores y que no logra trabajar de forma sistemática el tránsito hacia los estudios postsecundarios, viéndose así marginalizada la función de orientación vocacional. Ante esto aparece como urgente lograr el compromiso del equipo directivo, para incorporar la temática de orientación en la gestión de los liceos, lo que se debería reflejar 
en su institucionalización al interior de las unidades educativas. Al mismo tiempo, esto muestra que es necesario involucrar a más docentes en las tareas de orientación, de manera de promover un abordaje colectivo o de equipo, en el cual el orientador sea un articulador o gestor de políticas de orientación que serán implementadas en conjunto con otros miembros de la comunidad educativa.

- Se observó que los liceos en que se desarrolla este proceso no tenían incorporadas ciertas prácticas de gestión fundamentales para mejorar el trabajo de orientación. De lo anterior se desprende que, además de ganar espacio en la gestión global del liceo y formar equipos capacitados, se debería lograr que éstos sean capaces de formular un plan anual de orientación, ejecutarlo, realizar seguimiento periódico de sus acciones y evaluar resultados e impactos para retroalimentar su trabajo.

- Es necesario tomar en cuenta el contexto particular en que se está desarrollando el trabajo de asistencia técnica. Guichard señala que "las tareas en la orientación y la guía de carrera son respuestas prácticas a problemas que derivan del contexto social; o sea, son particulares en un contexto social dado" (Guichard, 2006: 2), por lo cual, para nutrir las prácticas de gestión mencionadas en el punto anterior con contenidos relevantes para los estudiantes, se debe tener claro que, dada la realidad socioeconómica en que se insertan estos liceos, no se está trabajando con estudiantes que mayoritariamente vayan a desarrollar "trayectorias lineales" (Oyarzún e Irrazabal, 2003) ${ }^{9}$. En ese sentido, además de incorporar información relativa a las áreas de conocimiento y carreras ofrecidas por el sistema de educación superior, deben incluirse posibilidades sobre inserción laboral, retorno económico y saturación de las carreras, así como acerca de becas, ayudas económicas y facilidades para estudiantes que trabajan. Los datos recogidos a través de una encuesta a estudiantes de tercero y

9 Los autores entienden por "trayectorias lineales" las caracterizadas por relaciones causa-efecto que cuentan con etapas claramente diferenciadas, las cuales nunca se sobreponen porque los ámbitos y espacios de la vida están socialmente organizados por distintas instituciones sociales. Así, un ejemplo de trayectoria lineal es la de aquel joven que, al salir de cuarto medio, se dedica exclusivamente a estudiar, o bien exclusivamente a trabajar, dejando atrás definitivamente el mundo de los estudios, sin combinar nunca ambas actividades. 
cuarto medio durante abril de 2008 nos muestran que, en ambos liceos, un porcentaje importante de jóvenes cree que seguirá lo que Oyarzún e Irrazabal (2003) definen como "trayectorias sincronizadas", entendidas como el desarrollo simultáneo de las actividades de estudio y trabajo.

Los gráficos 3 y 4 ponen en evidencia la primacía que tienen las trayectorias sincronizadas en las expectativas de los jóvenes de ambos liceos:

Gráfico No 3. ¿Qué crees que harás el año después de salir de cuarto medio? Respuestas alumnos Liceo Antonio Hermida, de Peñalolén (base: 70 alumnos).

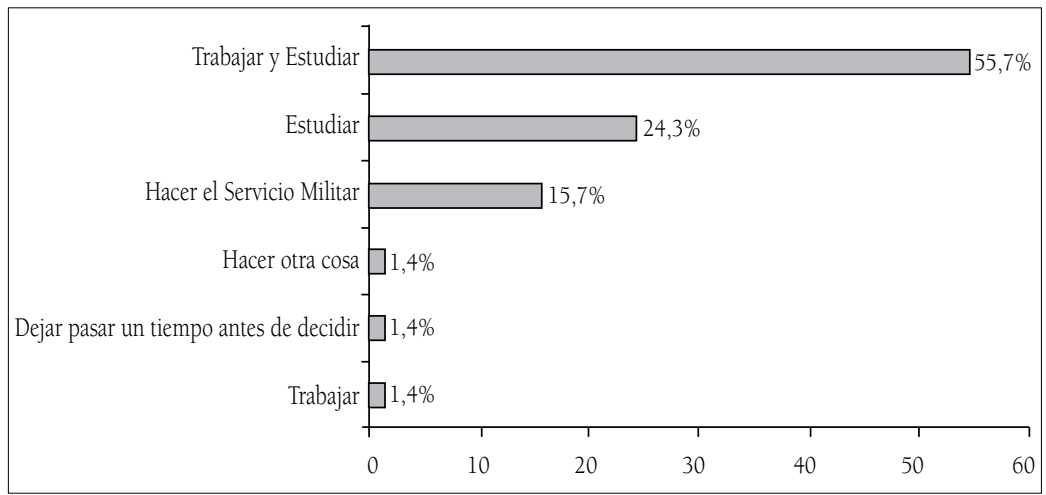

Gráfico No 4 . ¿Qué crees que harás el año después de salir de cuarto medio? Respuestas alumnos Liceo Municipal de Maipú (base: 250 alumnos).

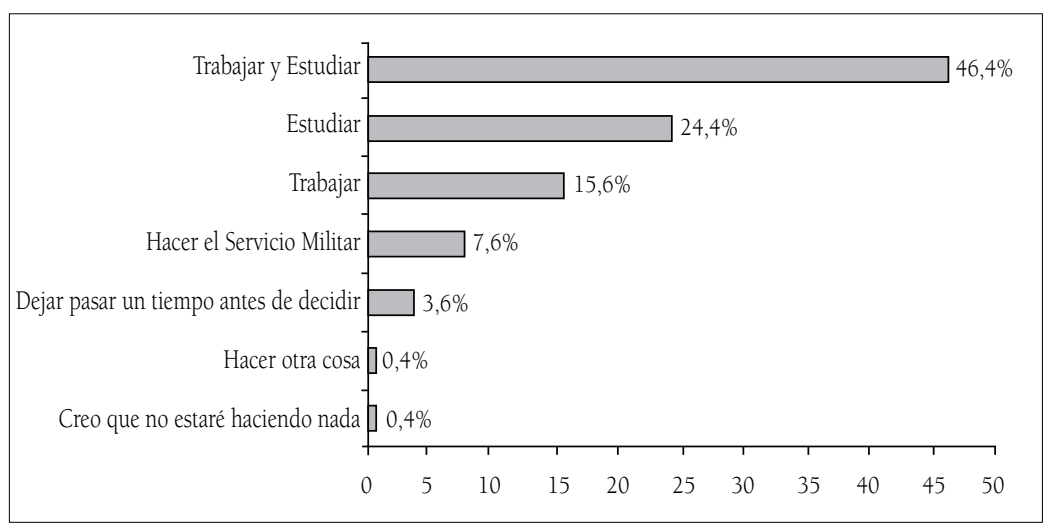


A partir del anterior diagnóstico, se definieron dos objetivos generales para esta iniciativa; el primero pensando en el proceso de asistencia técnica propiamente tal y el segundo correspondiente a la dimensión investigativa:

1) Implementar un proyecto piloto de apoyo a la orientación vocacional, entregando asistencia técnica a dos liceos municipales de estrato socioeconómico medio-bajo en la región metropolitana, para que estos puedan incorporar la orientación vocacional como componente relevante de su gestión escolar.

2) Conocer y comprender cómo los estudiantes secundarios de los liceos municipales escogidos atribuyen sentido y explican su tránsito desde la educación media a los estudios superiores y/o mundo del trabajo.

Dado que el objetivo general de este proyecto de asistencia técnica es la instalación de la orientación como un eje importante en la gestión escolar, la estrategia de acercamiento a los liceos apuntó a superar la sola interlocución de las orientadoras vocacionales, estableciendo vínculos de comunicación y trabajo con los equipos directivos de ambos liceos e intentando captar además el compromiso de otros docentes (preferentemente profesores jefes de cursos terminales).

\subsection{Primer año de implementación del proyecto de asistencia técnica en Maipú y Peñalolén}

Se han definido seis objetivos específicos para el logro del primer objetivo general asociado a la implementación del proyecto de asistencia técnica. A continuación se presentan dichos objetivos acompañados de las acciones realizadas durante 2008 para su logro. Cabe mencionar que este es un proyecto que sigue en curso durante 2009.

a) Legitimar al equipo de asistencia técnica del CSE como contraparte ante el equipo directivo de cada establecimiento.

Durante el segundo semestre de 2008 se desarrollaron reuniones con los equipos directivos de ambos establecimientos, en las que se expusieron los resultados de la encuesta diagnóstico de expectativas, diseñada y aplicada por el equipo de asistencia técnica del CSE a los alumnos y apoderados de tercer y cuarto año 
medio. La alta valoración que los equipos directivos de ambos liceos mostraron por este trabajo permitió estrechar la relación de colaboración entre los docentes directivos, que aportaban su experticia en materias pedagógicas, y el equipo de apoyo del CSE, que aportaba principalmente su conocimiento en metodologías de investigación y recolección de información.

b) Lograr un compromiso formal de parte del equipo directivo de cada liceo con el proyecto de apoyo a la orientación vocacional impulsado por el CSE

El equipo de asistencia técnica del CSE prestó apoyo a las orientadoras de ambos liceos en la definición de un plan anual de orientación que fue aceptado por ambos equipos directivos. Sin duda, el hecho de que ambas orientadoras formaran parte de los equipos directivos constituyó una ventana de oportunidades para la obtención de este apoyo.

c) Formar en cada liceo una masa crítica de docentes habilitados en orientación vocacional

Para el logro de este objetivo, mediante licitación pública, el CSE contrató los servicios de la Universidad Católica Silva Henríquez (UCSH), la que, a través de su Departamento de Educación, diseñó y dictó el curso de capacitación "Rol de la orientación vocacional en la gestión escolar: estrategias metodológicas para responder a los nuevos escenarios sociales y trayectorias juveniles". A éste asistieron las orientadoras y los profesores jefes de los terceros y cuartos años medios de ambos liceos.

d) Impulsar la formación de un equipo de orientación en cada liceo con la participación de la orientadora y al menos dos docentes capacitados en el curso desarrollado por el CSE y la UCSH.

Durante diciembre de 2008 se desarrollaron reuniones con los equipos de docentes capacitados en ambos liceos, con el fin de consolidar un grupo de trabajo que pudiera apoyar a la orientadora en la ejecución del plan anual de orientación que cada liceo desarrolle con la asistencia técnica del CSE durante 2009. 
e) Lograr que cada liceo formule su propio plan de acción en orientación vocacional, con una definición clara de objetivos, acciones asociadas a ellos, responsables de éstas e indicadores de resultados.

Como se señaló, el equipo de asistencia técnica del CSE apoyó a cada liceo en la formulación de un plan de trabajo durante 2008, el que, sin embargo, no contó con todas las características señaladas en el objetivo específico e), ya que se trató de planes de acciones con un horizonte más curricular -definición de unidades que se expondrían secuencialmente a lo largo del año- que estratégico (objetivos, acciones, indicadores). Queda, como desafío, desarrollar una planificación de horizonte estratégico durante 2009.

f) Instalar la práctica de seguimiento de trayectorias.

En la última reunión de evaluación del año escolar 2008 entre el equipo de asistencia técnica del CSE y el equipo directivo de cada liceo se acordó realizar un seguimiento a la promoción 2008 de cada liceo durante el primer semestre de 2009, la que sería ejecutada por los liceos con apoyo técnico del CSE.

\section{A modo de conclusión. Algunos aprendizajes del primer año de experiencia}

A partir de la experiencia acumulada en este primer año de trabajo se ha constatado que, si bien la labor y compromiso de los orientadores es fundamental para llevar a buen puerto un proyecto como éste, se hace indispensable involucrar desde un principio a los equipos directivos, ya que sólo éstos pueden y deben asignar un espacio significativo a la orientación vocacional en la gestión de los establecimientos. En este aspecto encontramos una de las primeras resistencias importantes a la idea ampliar el foco a la orientación como componente de la gestión escolar global, ya que en ambos establecimientos, luego de una reunión inicial con los directores, la total responsabilidad del proyecto fue delegada a las orientadoras, quienes durante todo el primer semestre fueron la única contraparte ante el CSE y las únicas ejecutoras del proyecto al interior de los establecimientos. Sólo en 
el segundo semestre de 2008 se concretaron las reuniones con los equipos directivos, los que gradualmente fueron incorporándose o, más bien, incorporando este proyecto de orientación dentro de su gestión.

Luego de un año de trabajo también se ha podido concluir que una práctica que debe promoverse es el diseño de un plan anual de orientación para cada liceo, en función de la realidad y necesidades de sus alumnos, que articule y otorgue sentido a esas tareas. Aunque en ambos establecimientos se logró diseñar una planificación anual que incorporó las necesidades de los alumnos, luego de haber realizado una encuesta diagnóstico, queda el desafío de realizar un plan en el cual se definan objetivos, estrategias para su consecución e indicadores para su evaluación, ya que la tendencia al interior de ambos establecimientos es desarrollar planificaciones de carácter curricular, en las que se definen unidades de orientación con contenidos que deben ser "pasados" a lo largo del año, pero sin un horizonte estratégico y evaluable claramente (más allá del chequeo de realización de cada unidad).

Es importante que, dado el carácter transversal que el actual marco curricular otorga a la orientación, se hace necesaria la voluntad y creatividad de los equipos directivos para introducir esta temática en otros espacios curriculares, lo que a su vez exige el compromiso y además la capacitación en el tema de otros profesores -además de los orientadores vocacionales-, situación abordada durante 2008 a través del curso dictado por la UCSH.

En ambos establecimientos es necesario implementar la práctica del seguimiento sistemático a los alumnos egresados. En uno de ellos no se había realizado antes debido a que en 2008 egresó su primera promoción de cuartos medios; en el otro, el seguimiento se limitaba a tomar nota de los puntajes en la PSU de los alumnos, más una consulta informal, vía telefónica, a algunos egresados, de la que además el orientador anterior no dejó registro.

En el contexto de este proyecto, se entiende por seguimiento una práctica institucionalizada, que tiene como meta contactar a 
todos los alumnos al año siguiente de su egreso de cuarto medio, registrando en una base de datos su situación académica y/o laboral. Esta tarea demanda el compromiso de los directivos del establecimiento para facilitar los recursos materiales, humanos y de tiempo que sean necesarios (horas para contactar a los egresados y completar bases de datos, líneas telefónica, computadores, entre otros). La información recolectada a través del seguimiento permitirá a los establecimientos contar con indicadores de gestión con los cuales evaluar y retroalimentar sus planes de orientación.

A través de las encuestas y en las conversaciones sostenidas con los jóvenes en el contexto de las actividades implementadas durante este primer año, se ha constatado que ellos esperan recibir, como parte del servicio de orientación vocacional de sus liceos, fundamentalmente información relativa al sistema de educación superior asociada al mercado laboral, tales como retorno económico de carreras, posibilidades de inserción laboral y saturación de carreras, e información y acompañamiento en la postulación a beneficios económicos que les ayuden a solventar sus estudios.

Finalmente, y tal vez una de las constataciones más relevantes en lo que va de esta experiencia, tanto en Peñalolén como en Maipú se ha observado que la mayoría de los jóvenes no está pensando en proseguir trayectorias lineales, vale decir, dedicarse exclusivamente a estudiar o bien incorporarse de manera definitiva al mundo laboral, sino que, por el contrario, visualizan en el futuro trayectorias sincronizadas que combinan estudio y trabajo.

Lo anterior plantea desafíos al sistema educacional en sus distintos niveles:

- Por una parte, la orientación vocacional entregada en los liceos debería apoyar a los estudiantes en el diseño de estrategias que les permitan seguir su formación terciaria, pero también insertarse en el mundo del trabajo con miras a sustentar sus estudios y, en muchos casos, solventar sus necesidades de subsistencia. En consecuencia, los equipos de orientación de los liceos deberían estar preparados para acompañar diversos tipos de trayectorias educativas y laborales, así como para habilitar a los jóvenes 
en la toma de decisiones en escenarios sociales de crecientes complejidad. Aun sin una normativa específica sobre orientadores, el marco de propósitos que ofrecen los Objetivos Transversales debiera constituirse en un referente para posicionar el tema de la transición a la educación superior desde el punto de vista de la formación integral del estudiante.

- Otra problemática observada en terreno a lo largo de este año indica que la evaluación que hacen las corporaciones municipales a sus liceos está asociada a logros en términos de puntaje PSU e ingreso a estudios universitarios, vale decir, sólo promueven incentivar las trayectorias lineales esperadas tradicionalmente de los jóvenes egresados de liceos científico-humanistas, lo que podría constituir una barrera de carácter institucional a un proyecto que incorpore necesidades diversas en materia de orientación y apoyo vocacional. Dicha práctica es del todo comprensible en el contexto nacional de fuerte valoración de los indicadores asociados al ingreso a la universidad, en detrimento de otras opciones. Ello debiera desafiar a los sostenedores de establecimientos secundarios a desarrollar indicadores de evaluación que recojan la diversidad de trayectorias educativas postsecundarias que siguen los jóvenes en la sociedad contemporánea.

- El carácter basal que adquieren las trayectorias sincronizadas en el marco de sistemas educacionales masificados como el chileno también plantea retos al sistema de educación superior: las instituciones de educación superior, ¿están considerando el nuevo perfil de jóvenes que se incorpora al sistema? Estas mismas instituciones, ¿generan condiciones para el adecuado desempeño académico de estudiantes que a la vez trabajan? Estas son sólo algunas de las interrogantes que se abren para estas instituciones en el actual escenario.

Los actores de los liceos y del CSE involucrados en esta experiencia de asistencia técnica saben que queda camino por recorrer; sin embargo, también han ido ganando conciencia de las fortalezas que poseen y que deben aprovechar para contribuir, desde su particular realidad, a formar estudiantes capaces de transitar a la educación terciara, con todas las oportunidades que ésta ofrece. 
La Secretaría Técnica del CSE agradece la colaboración de las corporaciones municipales de Maipú y Peñalolén, de los directores de liceo Juan Fuentes y Daniel Gutiérrez, así como de las orientadoras María Teresa Araneda y María Elena Cañas, cuya colaboración ha sido fundamental para el desarrollo de esta experiencia. También agradece la colaboración de Pablo Valdivieso en el diseño inicial de este proyecto.

\section{Referencias bibliográficas}

Consejo Superior de Educación (2007) Informe de gestión Gobierno Electrónico 2007, Etapas I, II, II, IV y V Reducida. Santiago de Chile: Secretaría Técnica del Consejo Superior de Educación.

De los Ríos, D. y Canales, A. (2007) "Factores explicativos de la deserción universitaria". Calidad en la Educación, 26.

Fernández, C., Peña, J., Viñuela, M. y Torío, S. (2007) Los procesos de orientación escolar y la toma de decisiones académica y profesional. Revista Complutense de Educación, 18(2).

Guichard, Jean (2006) Marcos teóricos para las nuevas tareas en orientación y guía sobre carreras. Orientación y Sociedad, 6.

James, Richard (2007) Students and student learning in mass systems of higher education: Six educational issues facing universities and academics leaders. Serie de seminarios Mass higher education in UK and International Contexts, Surrey.

Lagos, F. y Palacios, F. (2008) Orientación vocacional y profesional en colegios de bajo nivel socioeconómico: percepciones de orientadores y estudiantes. Calidad en la Educación, 28.

McDonough, Patricia M. (1997) Choosing Colleges: How social class and schools structure opportunity, State University of New York Press. Albany, Nueva York.

Oyarzún, A. e Irrazabal, R. (2003) Comportamiento de las trayectorias educacionales y laborales en jóvenes estudiantes. Última Década, 18.

Raczynski, D. y Muñoz, G. (2007) Reforma educacional chilena: el difícil equilibrio entre la macro y la micropolítica. Revista Electrónica Iberoamericana sobre Calidad, Eficacia y Cambio en Educación, V(3).

Sistema de Formación Permanente "Chilecalifica" (2008) Informe de Gestión 2003-2008. Línea de orientación vocacional y laboral. Gobierno de Chile: Ministerio de Economía, Ministerio de Educación, Ministerio del Trabajo y Previsión Social. 
Sistema de Formación Permanente "Chilecalifica" y MINEDUC (División de Educación General) (2008) Marco inicial para la institucionalización de la orientación escolar en la enseñanza Media. Sitio en internet. Disponible en: www.chilecalifica.cl/WebCMOrientacionWeb/ Documentos/7/1190385498884.doc

Recibido: 11 de mayo de 2009 Aceptado: 2 de junio de 2009 